\title{
Study of the Vascular Structure of Solid Breast Mass and the Predictive Value of Reversed Diastolic Flow in Predicting Malignancy
}

\author{
DINA M. MOHAMED, M.D. and AL-SHYMAA Z. MAHMOUD, M.D. \\ The Department of Radiodiagnosis and Medical Imaging, Faculty of Medicine, Tanta University, Tanta, Egypt
}

\begin{abstract}
Background: Breast cancer is considered the most common malignancy in females and the second most common leading cause of cancer death after lung cancer. Color, power and pulsating Doppler ultrasonography are more sensitive in the evaluation of solid breast mass depending on tumor new blood vessels growth that plays an important role in the growth of the malignant lesions.
\end{abstract}

Aim of Work: To study the vascular structure of solid breast mass and to detect the importance of reversed diastolic flow in detection of malignancy.

Material and Methods: This study included 114 solid breast mass in 100 female patients. Color Doppler ultrasound was done for all patients. We studied the blood vessels of the mass and we analyzed the wave pattern and detected the value of reversed diastolic flow in detecting malignancy.

Results: The vessels in malignant breast mass are of irregular caliber with central penetration while regular peripheral parallel vessels are suggestive of benignity also we found that reversed diastolic flow is significant sign of malignancy.

Conclusion: Color Doppler ultrasound is helpful to detect the vascular pattern of solid breast mass. Hypervascular mass with reversed diastole is suggestive of malignancy especially if the invading vessel is central and of irregular shape while presence of peripheral parallel vessels is suggestive of benignity however color Doppler indices such as high RI alone is not good indicator of malignancy.

Key Words: Breast cancer - Solid breast mass - Blood vessel - Color Doppler ultrasound.

\section{Introduction}

BREAST cancer is considered the most common malignancy in females and the second most common leading cause of cancer death after lung cancer [1]. With the development of breast ultrasonography and breast magnetic resonance imaging both complementary to mammography additional algorithms

Correspondence to: Dr. Dina M. Mohamed, The Department of Radiodiagnosis and Medical Imaging, Faculty of Medicine, Tanta University, Tanta, Egypt for diagnostic work up and screening high risk subgroups of women have emerged [2].

Mammography is considered the primary screening tool for cancer breast but its sensitivity is decreased with increasing the density of the breast tissue especially in young and lactating females. Using ultrasound was common after the introduction of the high frequency probes not only in the detection and characterization of lesions but also as guiding breast intervention procedures in the marking of lesion for surgery and needle biopsy [3].

Color, power and pulsating Doppler ultrasonography are more sensitive in the evaluation of solid breast mass depending on tumor new blood vessels growth that plays an important role in the growth of the malignant lesions $[\mathbf{3 , 4 , 5}$,

Color Doppler ultrasound is helpful to differentiate between a benign and malignant solid mass. Different Doppler characteristic such as location of signals, branching pattern of vessels and also resistive index (RI), pulsatility index (PI) and flow velocity nowadays can be detected $[4,5,6]$.

Many other methods are also used nowadays in the differentiation between benign and malignant breast solid masses such as contrast enhanced imaging techniques like dynamic contrast enhanced magnetic resonance imaging (DCE-MRI), contrast enhanced ultrasound (CEUS) and dynamic contrast enhanced computed tomography but all of these methods require a higher cost and operating skill level [7].

\section{Patients and Methods}

This study was performed between January 2016 and May 2017 in Tanta University Hospital. 
All patients signed on informed consent before the beginning of the study.

\section{Patients:}

One hundred female patients were included in this study and identified to have one or more solid breast masses. Patients age ranged between 17 and 60 years (mean age 35 years). Clinical examination, ultrasound and color Doppler were performed for each patient. All patients underwent biopsy (core or surgical biopsy).

Our exclusion criteria were patients with breast implants and patients with cystic breast lesions.

\section{Ultrasound examination:}

All sonographic studies were performed with the machine HITACHI and a high frequency probe of 7.5-10 MHZ to perform Color Doppler study.

I- The lesions were first imaged with grey scale ultrasound to show its character.

II- By using Color Doppler the vascular pattern of the lesion, number, shape and site of the blood vessels was detected.

III- We calculated the resistive index when vessels were detected within the lesion. $\mathrm{RI}=$ The maximum systolic velocity-minimum systolic velocity/maximum systolic velocity. A value for RI of 0.85 was used for the differentiation between benign and malignant lesions.

Slight pressure on the breast in cases of slow flow in blood vessels may be helpful. We use filter between 50 and $100 \mathrm{khz}$.

Histolo-pathological findings from surgery or core biopsy were used as gold references.

\section{Statical analysis:}

We used quantitative data presented by mean and range. The sensitivity, specificity, and positive predictive value of reversed diastolic flow were calculated to detect malignancy. A $p$-value of $<0.05$ was considered statistically significant.

\section{Results}

Patients and their final histopathological diagnosis:

This study included 100 patients with 114 solid breast lesions. Patients were classified into two groups according to the final histopathological results: Benign (60 lesions) and malignant (54 lesions). The final histopathological analysis showed that $72 \%$ of the benign lesions were fibroadenoma while $84 \%$ of the malignant lesions were invasive ductal carcinoma (Table 1).
Comparison of the color Doppler findings and the Resistive index in both groups:

We compared the Color Doppler findings of all breast lesions in both groups. Vascularity was detected in 24 of 60 benign masses $(40 \%)$ and in 45 of $54(83 \%)$ malignant masses. The vessels in benign mass were peripheral and the vein and artery were parallel to each other while $77 \%$ of the malignant lesions showed invading arteries in the center of the lesion. The resistive Index was calculated. It ranged between 0.4 and 1.30 (mean 0.85 ) in malignant lesions and from 0.25 to 1 (mean 0.62 ) in benign lesions with significant difference $(p<0.05)$ (Table 2).

Shape of vessels in both groups of breast lesions:

The vessels in benign masses were generally of regular shape representing $91 \%$ while $70 \%$ of the vessels in malignant lesions were tortuous or irregular (Table 3).

The diagnostic value of color Doppler ultrasound and reversed diastole in detecting malignancy:

We detected reversed diastolic flow in 10 out of 45 malignant lesions (22\%) which showed a high grade of malignancy in histopathological analysis with sensitivity of $94 \%$, specificity of $96 \%$ and a positive predictive value of $95 \%$.

Some selected cases demonstrated in Figs. (14 ), each figure represent one case.

Table (1): Patients and final pathological diagnosis.

\begin{tabular}{lc}
\hline Diagnosis & $\begin{array}{c}\text { No. of breast } \\
\text { lesions }\end{array}$ \\
\hline Age (years): & 35 \\
Mean & $17-60$ \\
Range & \\
Histopathological analysis: & $43(0.38 \%)$ \\
Fibroadenoma & $08(0.07 \%)$ \\
Intra-mammary lymph node & $04(0.04 \%)$ \\
Intraductal Papilloma & $05(0.04 \%)$ \\
Phyllodes tumor & $45(0.40 \%)$ \\
Invasive ductal carcinoma & $02(0.02 \%)$ \\
Ductal carcinoma in situ & $01(0.01 \%)$ \\
Lobular breast carcinoma & $01(0.01 \%)$ \\
Metaplastic tumor & $01(0.01 \%)$ \\
Anaplastic carcinoma & 114 \\
\hline Total &
\end{tabular}


Table (2): Color Doppler findings and RI in both groups.

\begin{tabular}{lcccccc}
\hline Pathology (No.) & $\begin{array}{c}\text { Hypervascular Avascular } \\
\%\end{array}$ & Total & $\begin{array}{c}\text { RI } \\
\text { (mean) }\end{array}$ & $\begin{array}{c}\text { R1 } \\
\text { (range) }\end{array}$ & $\begin{array}{c}p \text { - } \\
\text { value }\end{array}$ \\
\hline Benign Lesions & $24(40 \%)$ & $37(60 \%)$ & 60 & 0.62 & $0.25-1$ & $p<0.05$ \\
Malignant Lesions & $46(85 \%)$ & $8(15 \%)$ & 54 & 0.85 & $0.4-1.32$ & \\
\hline
\end{tabular}

Table (3): Shape of vessels in both groups.

\begin{tabular}{lccc}
\hline & $\begin{array}{c}\text { No. Benign Vascular } \\
\text { Masses on } \\
\text { Color Doppler } \%\end{array}$ & $\begin{array}{c}\text { No Malignant } \\
\text { Vascular Masses on } \\
\text { Color Doppler\% }\end{array}$ & $\begin{array}{c}p \text { - } \\
\text { value }\end{array}$ \\
\hline Regular shape & $21(91 \%)$ & $14(30 \%)$ & $p<0.05$ \\
Tortuous and or Irregular shape & $2(9 \%)$ & $31(70 \%)$ & \\
\hline Total & 23 & 45 & \\
\hline
\end{tabular}
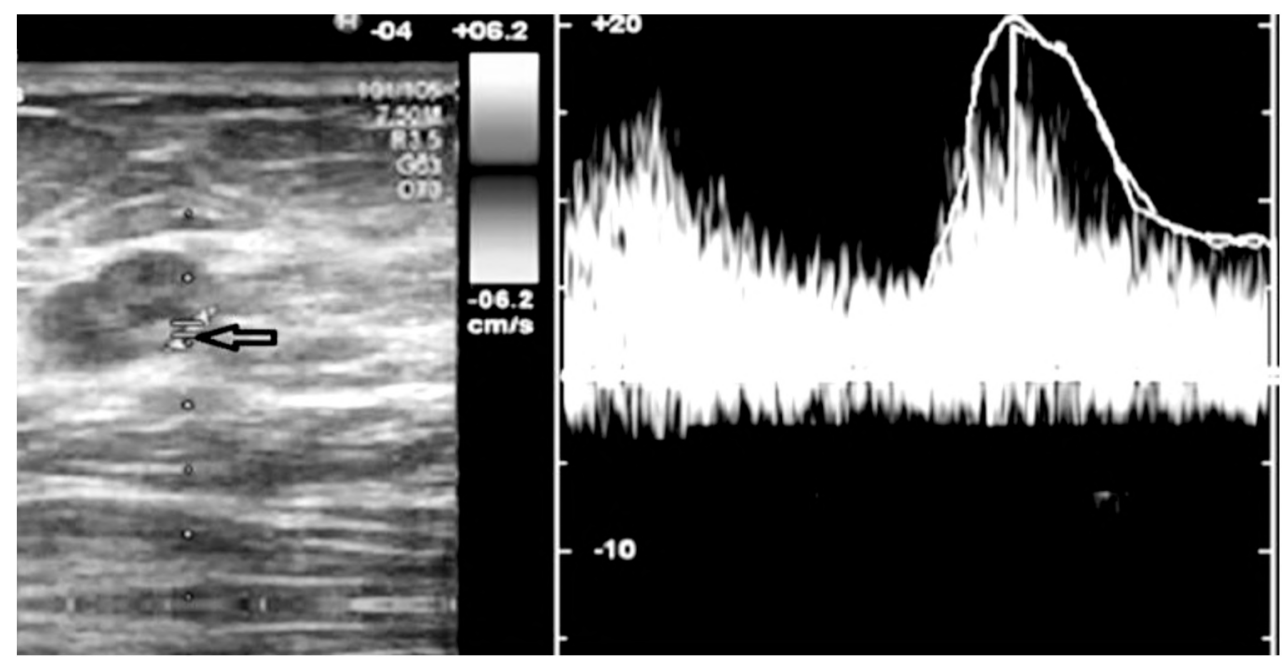

Fig. (1): A female patient aged: 40 years old presented by right breast mass. B mode shows the mass is well defined hypoechoic and lobulated. Color Doppler ultrasound shows the blood vessels are peripheral and parallel to each other (arrow). Spectral wave analysis shows: High diastolic flow with RI: 0.69. Histopathological diagnosis: Papilloma.

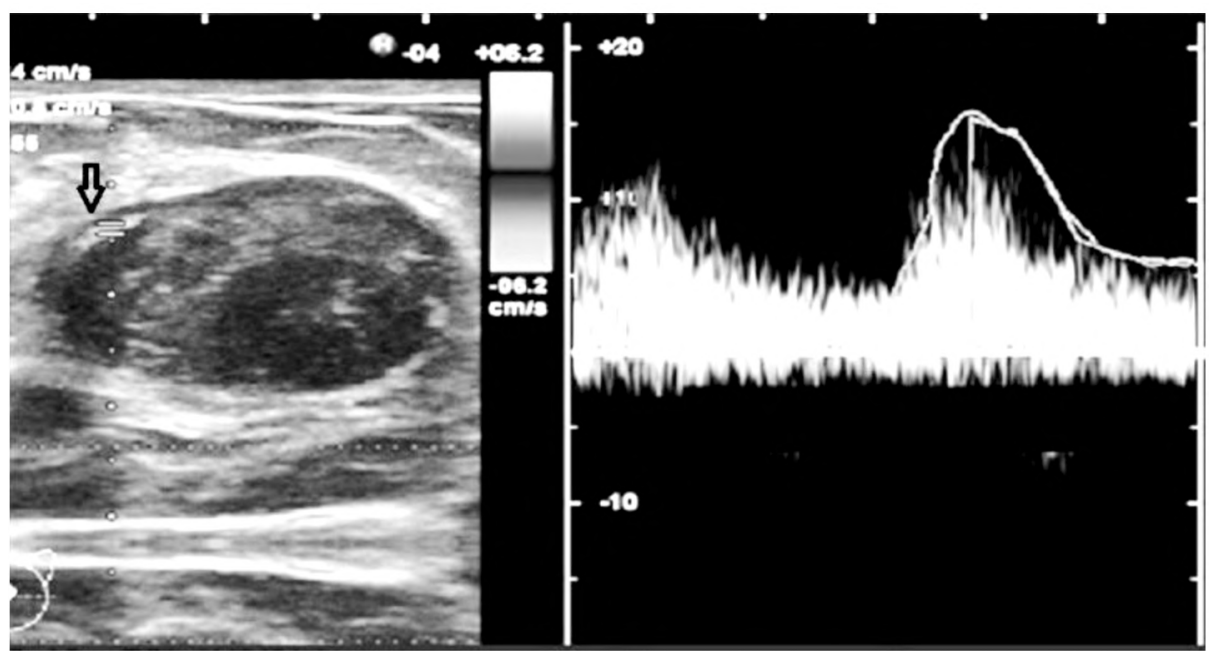

Fig. (2): A female patient aged 45 years represented with left breast mass. B mode shows the mass is well defined oval and hypoechoic. Color Doppler ultrasound shows the blood vessels are peripheral and parallel to each other (arrow). Spectral wave analysis shows normal diastolic flow with RI: 0.55. Histopathological diagnosis: Fibroadenoma. 


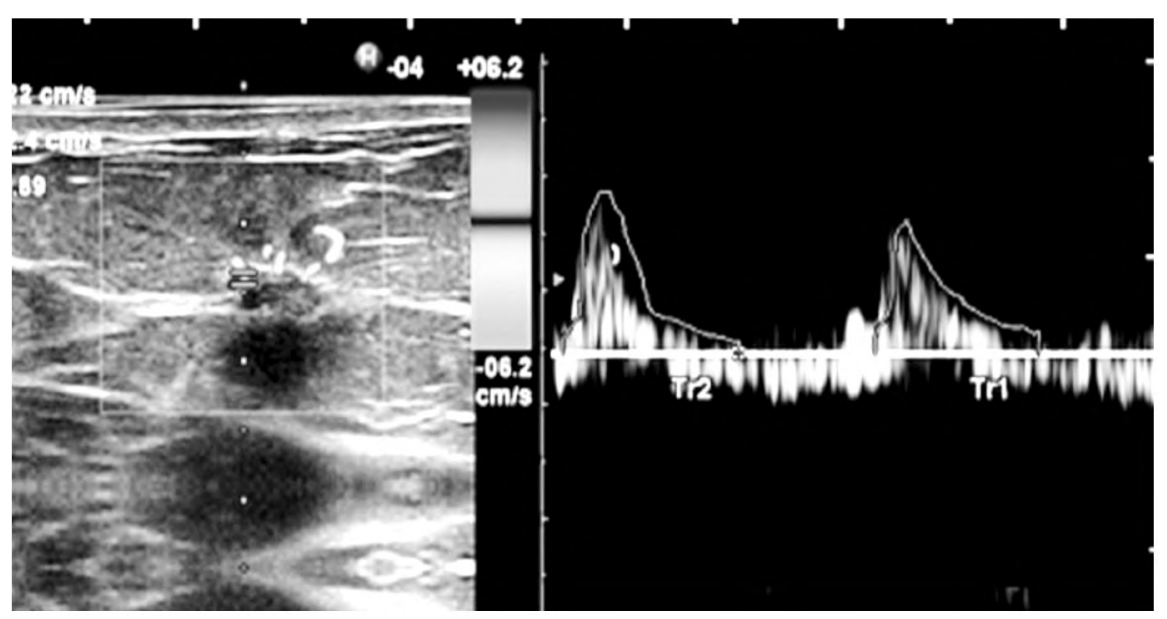

Fig. (3): A female patient aged: 55 years old presented by left breast mass. B mode shows the mass is ill defined and hypoechoic. Color Doppler ultrasound shows the blood vessels are irregular penetrating the mass .Spectral wave analysis shows reversed diastolic flow with RI: 0.89. Histopathological diagnosis: Colloid carcinoma.

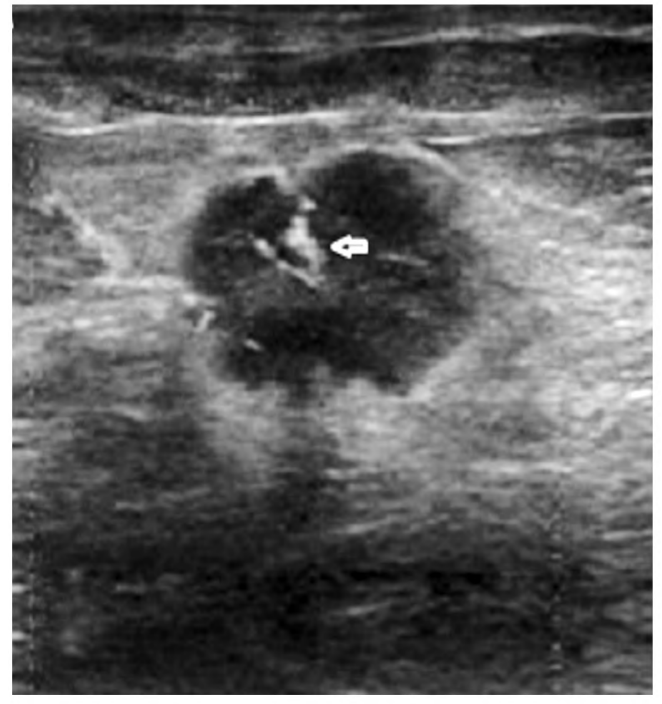

(A)

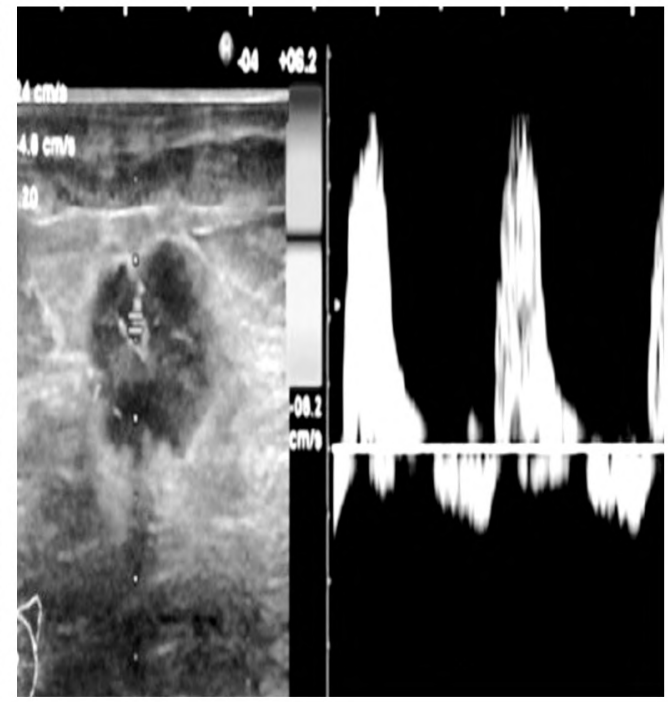

(B)

Fig. (4): A female patient aged 56 years old presented by left breast mass. (A) B mode shows ill-defined speculated hypoechoic mass color Doppler shows the blood vessels are irregular central and penetrating the mass (arrow). (B) Spectral wave analysis shows reversed diastolic flow with RI 1.2. Histopathological diagnosis: Invasive duct carcinoma.

\section{Discussion}

Malignant breast lesions need blood vessels for growth. A procedure such as color Doppler ultrasound has the ability to visualize the blood vessels within solid lesion. The aim of this study was designed to determine the value of Color Doppler sonography in study of the vascular structure of solid breast mass and to detect the importance of reversed diastolic flow in the detection of malignancy.

The main finding of this study was malignant breast lesions are more vascular than benign lesions. In our study hypervascularisation was detected in
$80 \%$ of malignant breast masses and only in $7 \%$ of benign masses.

Gupta et al., [8] in their study which included 173 solid breast masses in 148 patients found that hypervascular breast mass with tortuous irregular blood vessels and presence of a central penetrating vessel significantly associated with malignancy. Seventy-seven percent of malignant breast masses showed hypervascularisation when compared to the surrounding tissue but only $8.26 \%$ benign masses only were hypervascular.

Hypervascular breast lesion increases the probability of malignancy but avascularity on Color 
Doppler ultrasound should not be interpreted as a sign of benignity $[9,10]$. In this study $16.6 \%$ of malignant lesions were avascular on color Doppler. This finding may be due to the small vessels within the mass. A solid mass with central blood vessels is suspicious to be malignant. The presences of penetrating vessels in the mass directly is suggestive of malignancy [9]. In this study, $77 \%$ of the malignant lesions show arteries in the center of the lesion.

A high RI is associated with a high chance of malignancy this may be due to areas of blood vessels stenosis, occlusions, arteriovenous shunts and lack of smooth muscle [9].

It has been reported that the analysis of Doppler indices such as RI and PI is helpless to differentiate between benign and malignant breast lesions [11,12] because there is an overlap of these indices. Although malignancies are showed to have a high Doppler indices RI cannot be a definite sign to differentiate between benign and malignant lesions [9].

Gupta et al., [8] found in their study that the 43 vascular malignant lesions had a mean RI value of 0.80 with a SD of 0.16 (range of 0.50-1.02).

Davoudi et al., [10] reported the mean values of RI in benign lesions and malignant lesions were $0.65 \pm 0.065$ (range of $0.52-0.89$ ) and $0.71 \pm 0.093$ (range, 0.57-0.75). In our study RI values range between 0.4 and $1.30 \mathrm{~m}$ (mean 0.85 ) in malignant lesions higher than in benign lesions which ranges from 0.25 to 1 (mean 0.62 ).

Both benign and malignant solid breast masses show vascularity but in malignant mass vascularity is owed to new blood vessels formation which are tortuous of irregular course with straight or curved capsular blood vessels and regular caliber are predictors of benignity [8,9] .

The study by Gupta et al., [8] showed that the blood vessels in benign lesions were of regular shape and caliber with regular branching. Thirtyone of malignant lesions (72\%) showed tortuous arteries with irregular caliber arranged in a chaotic pattern. The vessels in benign masses in this study were generally of regular caliber with regular branching representing $91 \%$ while $70 \%$ of vessels in malignant lesions were tortuous or irregular.

Ibrahim et al., [13] in their study which included 91 women with 102 breast lesions ( 55 benign, 47 malignant). Thirty six out of 47 malignant lesions showed central blood vessels. Sensitivity and spe- cificity of the presence of central pretreating artery in detecting malignancy were $76.5 \%$ and $80.0 \%$. In this study $77 \%$ of the malignant breast lesion showed central penetrating artery.

Eleonora et al., [14] in their study which included 1074 breast masses showed parallel artery and vein sign was identified in 142 benign masses with a specificity of $99.3 \%$ and concluded that although this sign is not of the common findings it showed 99\% positive predictive value in the detection in benign breast lesion wherever it seen in the capsule or inside the mass. Ninety percent of the benign breast lesions in this study showed this sign (54 out of 60 benign lesions).

The absence or reversal of diastole on spectral Doppler is a very specific sign for predicting malignancy in solid breast masses. The disappearance or reversal of vascular flow represents an extreme increase of peripheral resistance of tumoral vessels due to previously mentioned phenomenon of stenosis and occlusions indicative of malignant vascularity [9].

We found 10 out of 45 malignant lesions (22\%) showed reversed diastolic flow. Some of these lesions showed a high RI measured 1 which showed a high grade of malignancy in histopathological analysis with sensitivity of $94 \%$, specificity of $96 \%$ and a positive predictive value of $95 \%$.

In the study of Gupta et al., [8] 8 out of 43 malignant masses showed reversed/absent diastolic flow and 7 of these lesions showed a high grade of malignancy in histopathological analysis. They reported that this sign was not seen in any benign masses with a specificity of $99 \%$ and a positive predictive value of $97 \%$.

There are some limitation in this study first, small number of patients. Secondly, color Doppler ultrasound is operator dependent.

From our study we concluded that color Doppler ultrasound is helpful to detect the vascular pattern of a solid breast mass. A hypervascular mass with reversed diastole is suggestive of malignancy especially if the invading vessel is central and of irregular shape while the presence of peripheral parallel vessels is suggestive of benignity. However, color Doppler indices such as a high RI alone is not good indicator of malignancy which make histolopathological analysis necessary beside the color Doppler ultrasound.

\section{Conflict of interest:}

We have no conflict of interest. 


\section{References}

1- MANSOUR G.: Vascularity of breast lump, Open Journal of Obstetrics and Gynecology, 3: 659-62, 2013.

2- BONNIE N.J. and EDWARD A. STICKLES: The evaluation of breast imaging: Past to present, Radiology, 273 . 2S: S23-S44, 2014.

3- MILZ P., LIENEMANN A., KESSLER M. and REISER M.: Evaluation of breast lesions by power Doppler sonography. Eur. Radiol., 11: 547-54, 2001.

4- LEE S.H., CHOI H. and BACK S.: Role of color and power Doppler imaging in differentiating between malignant and benign solid breast masses, Journal of Clinical Ultrasound, 30 (8): 459-64, 2002.

5- BATOOL S., SHANZAT A. and AHSAN Z.: Doppler evaluation of breast solid masses differentiation of benign and malignant masses, Role of RI. Annals, 12 (2): 32526, 2006.

6- BHATTI B., HELVIE M. and CASON P.: Discrimination of sonography detected breast mass masses using frequency shift colour Doppler imaging in combination with age and grey scale criteria, J. Ultrasound Med., 20 (4): 343 50, 2001.

7- MA Y., LI G., LI J. and REN W.: The diagnostic value of super b microvascular imaging (SMI) in detecting blood flow signal of breast lesions, Medicine, 94 (36): 1502-7, 2015.
8- GUPTA K., CH T. and M.K.: Role of Color Doppler for Assessment of Malignancy in Solid Breast Masses: A Prospective Study, International Journal of Anatomy, Radiology and Surgery Jan., Vol-6 (1): RO59-RO65, 2017.

9- STANZANI D., CHALA L. and BARROS N.: Can Doppler or contrast-enhanced ultrasound analysis add diagnostically important information about the nature of breast lesions? Clinics (Sao Paulo), 69 (2): 87-92, 2014.

10- DAVOUDI Y., BORHANI B. and RAD M .: The role of Doppler sonography in distinguishing malignant from benign breast lesions. Journal of Medical Ultrasound, 22 (2): 92-95, 2014.

11-YANG W. and DEMPSEY P.: Diagnostic breast ultrasound: current status and future directions. Radio. Clin. N. Am., 45: 845- 61. 2007.

12- SCHROEDER R.J., BOSTANJOGLO M. and RADEMAKER J.: Role of power Doppler techniques and ultrasound contrast enhancement in the differential diagnosis of focal breast lesions. Eur. Radiol., 13 (1): 68-79, 2003.

13- IBRAHIM R., RAHMAT K. and FADZLI F.: Evaluation of solid breast lesions with power Doppler: Value of penetrating vessels as a predictor of malignancy. Singapore Med. J. Nov., 57 (11): 634-640, 2016.

14- ELEONORA H., CLAUDIO S. and GERARDO: Parallel Artery and Vein: Sign of Benign Nature of Breast Masses.American Journal of Roentgenology, 198: W76-W82, 2012.

\title{
دراسة الأوعية الدموية لأورام الثدى الصلبة والقيمة التنبؤية لعكس التدفق الأنبساطى لتوقع الورم الخبيث الواليث
}

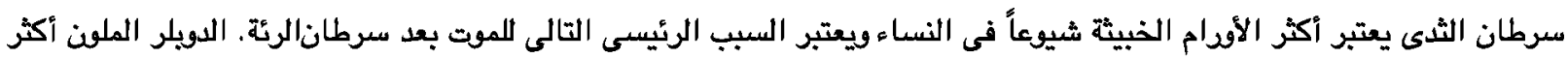

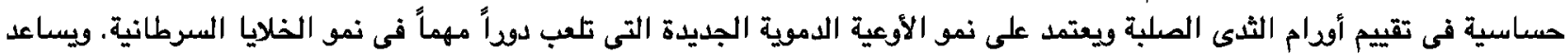 \\ الدوبلر الملون في تحديد هذه الأومية الجديدة.

